\title{
Directed flow of D mesons at RHIC and LHC energy within a transport approach: non-perturbative dynamics, vorticity and electromagnetic fields
}

\author{
Salvatore Plumari ${ }^{1,2, *}$, Lucia Oliva ${ }^{3}$, Yifeng Sun $^{2}$, and Vincenzo Greco ${ }^{1,2}$ \\ ${ }^{1}$ Department of Physics and Astronomy 'E. Majorana', University of Catania, Via S. Sofia 64, I-95123 \\ Catania \\ ${ }^{2}$ INFN-Laboratori Nazionali del Sud, Via S. Sofia 62, I-95123 Catania, Italy \\ ${ }^{3}$ Institut für Theoretische Physik, Johann Wolfgang Goethe-Universität, Max-von-Laue-Str. 1, 60438 \\ Frankfurt am Main, Germany
}

\begin{abstract}
We study the propagation of charm quarks in the quark-gluon plasma (QGP) by means a relativistic Boltzmann transport (RBT) approach coupled to electromagnetic field. The interplay between these fields is responsible to generate large rapidity odd directed flow $v_{1}$ of $\mathrm{D}$ mesons and for a large splitting of directed flow $\Delta v_{1}$ between neutral $\mathrm{D}$ and anti-D mesons. We show that the large $v_{1}$ is generated by the longitudinal asymmetry between the bulk matter and the charm quarks and by a large non-perturbative interaction in the QGP medium.
\end{abstract}

\section{Introduction}

Charm quarks are the earliest charged particles appearing in the ultra relativistic Heavy Ion collisons (uRHICs). Due to their short formation time with $\tau \approx 0.1 \mathrm{fm} / \mathrm{c}$ and a thermalization time which is comparable to the QGP lifetime they are good probes of both the initial stage and the subsequent evolution into a thermalized QGP. The matter produced in uRHICs is not only in the regime of extreme temperature and density but also in the regime of large vorticity due to huge initial angular momentum and intense electromagnetic (e.m.) fields that are the two possible sources for a finite directed flow $v_{1}$. Recent theoretical studies have shown that the heavy quarks can manifest a large directed flow which is one order of magnitude larger than the one of light hadrons $[1,2]$. This was predicted for the average directed flow of D mesons [2] and was predicted for the first time a splitting in the directed flow between $D^{0}$ and $\overline{D^{0}}$ mesons induced by e.m. field [1]. In these first theoretical investigations a Langevin approach coupled to the Maxwell equations have been used. Recent experimental data from STAR collaboration [3] at top RHIC energies and from ALICE collaboration [4] at LHC energies have shown a directed flow larger than the one of light charged hadrons [5]. Recently, in Ref.s [6, 7], important features of the directed flow splitting $\Delta v_{1}$ induced by e.m. fields have been found suggesting that measurement of $\Delta v_{1}$ of $\mathrm{D}$ mesons and leptons form $Z^{0}$ decay and their correlations could be powerful tool to probe the initial e.m. fields in uRHICs.

\footnotetext{
*e-mail: salvatore.plumari@dfa.unict.it
} 


\section{Charm quarks transport equation in the electromagnetic field}

We discuss results on the directed flow obtained within a relativistic Boltzmann transport approach developed to study the dynamics of heavy-ion collisions and recently extended to describe anisotropic flows $v_{n}$ and the nuclear modification factor $R_{A A}$ of $D$ mesons [8-13]. The dynamical evolution of the QGP bulk (gluons and light quarks) as well as of charm quarks in the QGP bulk is described by the following equations:

$$
\begin{aligned}
{\left[p_{\mu} \partial_{x}^{\mu}+q_{j} F_{\mu v}(x) p^{v} \partial_{p}^{\mu}\right] f_{j}(x, p) } & =C_{22}\left[f_{j}, f_{k}\right](x, p) \\
{\left[p_{\mu} \partial_{x}^{\mu}+q_{Q} F_{\mu \nu}(x) p^{v} \partial_{p}^{\mu}\right] f_{Q}(x, p) } & =C_{22}\left[f_{j}, f_{k}, f_{Q}\right](x, p)
\end{aligned}
$$

where $f_{j, k}(x, p)$ is the phase-space distribution function of quarks and gluons $j, k=g, q, \bar{q}$ while $f_{Q}(x, p)$ is the phase-space distribution function of charm quarks $Q=c, \bar{c}$. In the above equations the $F_{\mu \nu}$ is the electromagnetic strength tensor of the external e.m. field. On the right-hand sides $C_{22}$ is the collision integral accounting for $2 \rightarrow 2$ scattering processes. We have employed a bulk with massive quarks and gluons given by a Quasi-Particle Model (QPM) [14] where quarks and gluons have a thermal masses $m_{g, q}(T) \propto g(T) T$ tuned to reproduce the lattice QCD thermodynamics [15]. The charm quarks interact with the bulk by means of $2 \rightarrow 2$ elastic scattering, see [12] for details of the approach used.

The e.m. field included in the transport equations are computed following Ref. [16] and the results shown have been obtained assuming a constant electrical conductivity $\sigma_{e l}$ of the QGP. The values of $\sigma_{e l}$ used are in agreement with the lattice QCD calculations [17] and correspond to typical values that can be explored in uRHICs. The total e.m. field produced by the two nuclei is obtained solving the Maxwell equations. The approach consists first to calculate the elementary e.m. fields generated by single point-like charges $e$ located in the transverse plane and moving towards $+z$ and $-z$ respectively with speed $\beta$ and then these field are convoluted with the transverse charge distribution of the nuclei $\rho$ to get the total e.m. field. In Fig. 1 it is shown the time evolution of e.m. field for two different collision energies
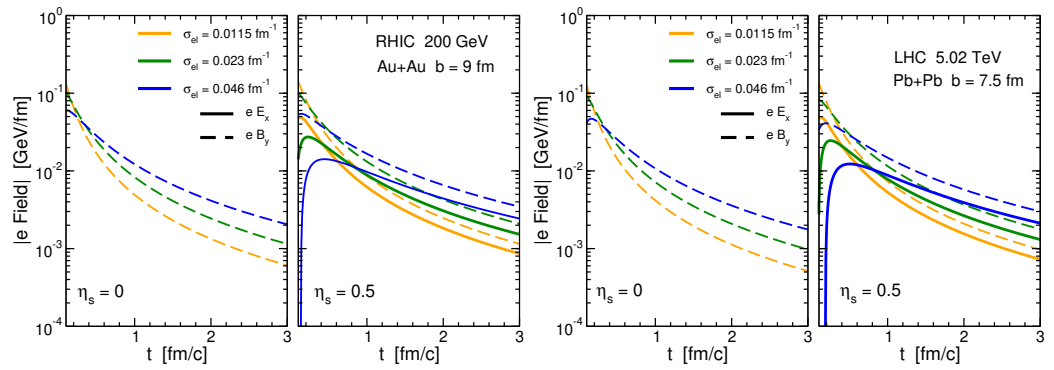

Figure 1. Time evolution of $\left|e E_{x}\right|$ (solid curves) and $\left|e B_{y}\right|$ (dashed curves) in Au+Au collisions at $\sqrt{\sigma_{N N}}=200 \mathrm{GeV}$ with $b=9 \mathrm{fm}$ (left panel) and $\mathrm{Pb}+\mathrm{Pb}$ collisions at $\sqrt{\sigma_{N N}}=5.02 \mathrm{TeV}$ with $b=7.5$ $\mathrm{fm}$ (right panel). The different colours correspond to different values of the electric conductivity $\sigma_{e l}$.

at top RHIC energies (left panel) and LHC energies (right panel). The curves are for two values of the space-time rapidity: $\eta_{s}=0$ and $\eta_{s}=0.5$ and for different values of the electric conductivity as computed in lattice QCD [17]. As shown, at mid-rapidity $\eta_{s}=0$ the electric field vanishes due to symmetry while at forward and backward rapidity $E_{x}$ become huge and comparable to $B_{y}$. The $\left|e B_{y}\right|$ decrease by one order of magnitude in less than $1 \mathrm{fm} / c$ and the decrease is stronger for smaller electric conductivity. On the other hand, at larger $\eta_{s}$ the time evolution of the $\left|e B_{y}\right|$ becomes milder. 
In coordinate space the bulk it has been initialized by using the standard Glauber model assuming boost invariance along the longitudinal direction. In momentum space we have considered a mixture of Boltzmann-Jüttner distribution function up to transverse momentum $p_{T}^{m j}=2 \mathrm{GeV}$ at RHIC $\left(p_{T}^{m j}=3.5 \mathrm{GeV}\right.$ at LHC) while mini-jet distributions as calculated by pQCD at NLO order in [18] at higher transverse momentum. The charm quark distributions are initialized in coordinate space by using $N_{\text {coll }}$. In momentum space we use the charm quark distribution according to the Fixed Order + Next-to-Leading Log (FONLL) calculation [19]. In a non-central collision, the system of the two incoming nuclei possesses an angular momentum $J$ which depends on the collision energy and impact parameter. After the collision a fraction of the angular momentum is transferred to the plasma created which manifests in a nonzero vorticity of the system. In hydrodynamical simulations $J$ has been introduced as an asymmetric initial energy density distribution in the longitudinal direction [20, 21]. We show the results obtained in [22] where similar initial conditions have been implemented in a transport approach in order to take into account the nonzero angular momentum. We show
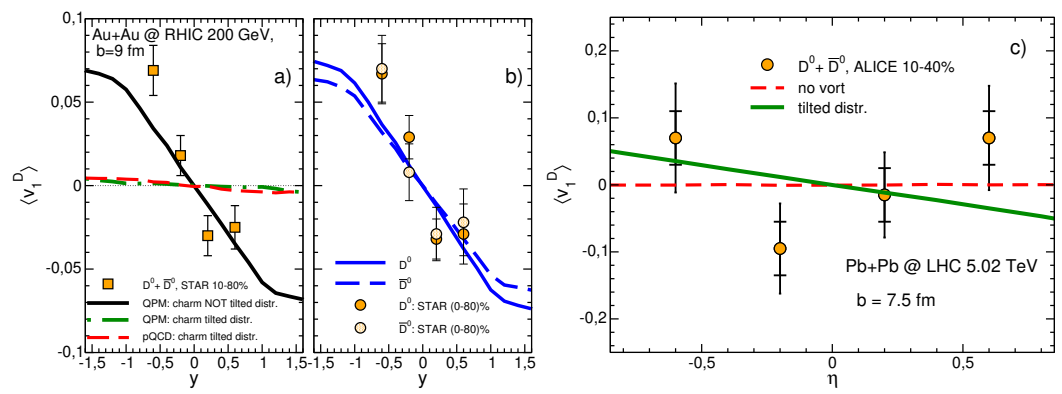

Figure 2. $v_{1}(y)$ of D mesons as a function of rapidity at RHIC (left) and LHC (right). In panel a) the black solid line is the case where bulk is tilted but charm distribution not while the green dot-dashed is the case where charm quark are distribuited with a tilted distribution like the bulk. The red dashed line corresponds to the case where charm quarks interact according to a pQCD interaction. In panel b) it is shown the effect of the e.m. field on the $v_{1}$ of $D^{0}$ meson (blue solid line) and $\overline{D^{0}}$ meson (blue dashed line). The experimental data are taken from [3]. In panel c) the solid green line is the case where the bulk has a tilted distribution while the red dashed line is the case without vorticity with a bulk forward and backward symmetric. The experimental data are taken from [4].

how the rapidity dependence of the directed flow $v_{1}$ of neutral $D$ mesons is originated by the vorticity of the bulk matter coming from the initial tilted longitudinal distribution and the non-perturbative interaction of charm quarks. As shown in Fig. 2 a) if the initial charm quarks are initialized according to a tilted distribution, as done for the bulk, the large $v_{1}$ of the charm quarks disappears and the final $v_{1}$ is very small, see green dot-dashed lines shown in Fig. 2 a). On the other hand, the large $v_{1}$ shown by the black solid line is due to a pressure gradient of the bulk to the charm quarks toward the negative $x$-direction at positive $\eta_{s}$. The effect of this pressure gradient comes from the non-perturbative interaction of the HQ with the bulk. In fact, if one keeps the non-tilted space distribution but assume a drag and diffusion of the charm quarks according to pQCD interaction one get a very small $v_{1}$ as shown by the red dashed line in Fig. 2 a). The charm quark develops a finite $v_{1}$ only if the initial distribution of charm quark is not tilted and charm quarks interact non-perturbatively with an interaction coming from QPM. However, in addition to this dynamics there is also a motion induced by the e.m. field produced in the collision. In Fig. 2 b) it is shown the effect of the e.m. fields on the directed flow of $\mathrm{D}$ mesons for $\mathrm{Au}+\mathrm{Au}$ collisions at top RHIC energy. The value of the electric conductivity used is $\sigma_{e l}=0.023 \mathrm{fm}^{-1}$ which is the value from lattice QCD calculation 
at $T=2 T_{c}$. As shown in Fig. 2 b) at forward rapidity positively-charged particles get a negative contribution to the $v_{1}$ and negatively-charged particles get a positive contribution to the $v_{1}$. This suggest that the overall effect is slightly dominated by the electric field. Finally, in Fig. 2 c) the results for D meson are shown in comparison with the experimental data at LHC energies. The dashed line correspond to the standard initialization while the solid line is the result with tilted initial distribution for the bulk where both simulations includes the e.m. fields. The ALICE data indicate a positive slope for the combined directed flow of D meson that is smaller than the one observed at RHIC.

\section{Conclusion}

The directed flow $v_{1}(y)$ of charm quarks it has been studied within a transport approach at both RHIC and LHC energy. We have observed that the origin of the directed flow for the $D$ meson comes from two process: i) the rotation induced by the initial angular momentum of the spectators transferred to the bulk matter which produce a $v_{1}(y)$ of $D^{0}$ and $\overline{D^{0}}$ mesons several times larger than the one of the light mesons and ii) the interaction of HQ with the bulk matter which is non-perturbative and is responsible for the transfer of the gradient pressure of the bulk matter to the charm quarks. We have studied the splitting of $v_{1}(y)$ of $\mathrm{D}$ mesons due to the e.m. field. It has been shown that this effect is in agreement with the experimental data at RHIC energy, but due to large error bars currently it is not possible to draw any conclusions. At LHC energy theoretical models based on electromagnetic field that assume a medium at constant electric conductivity are not able to account for it.

\section{References}

[1] S.K. Das et al., Phys. Lett. B 768, 260 (2017).

[2] S. Chatterjee, P. Bożek, Phys. Rev. Lett. 120, 192301 (2018).

[3] J. Adam et al. (STAR), Phys. Rev. Lett. 123, 162301 (2019).

[4] S. Acharya et al. (ALICE), Phys. Rev. Lett. 125, 022301 (2020).

[5] L. Oliva, Eur. Phys. J. A 56, no.10, 255 (2020).

[6] Y. Sun, S. Plumari, V. Greco, Phys. Lett. B 816, 136271 (2021).

[7] Y. Sun, S. Plumari, V. Greco, Eur. Phys. J. C 80, 16 (2020).

[8] M. Ruggieri, F. Scardina, S. Plumari, V. Greco, Phys. Lett. B 727, 177 (2013).

[9] S. Plumari, G.L. Guardo, F. Scardina, V. Greco, Phys. Rev. C 92, 054902 (2015).

[10] M. Ruggieri et al., Phys. Rev. C 92, 064904 (2015).

[11] S.K. Das, M. Ruggieri, F. Scardina, S. Plumari, V. Greco, J. Phys. G 44, 095102 (2017).

[12] F. Scardina et al., Phys. Rev. C 96, 044905 (2017).

[13] S. Plumari et al., Phys. Lett. B 805, 135460 (2020).

[14] S. Plumari, W.M. Alberico, V. Greco, C. Ratti, Phys. Rev. D 84, 094004 (2011).

[15] S. Borsanyi et al., JHEP 11, 077 (2010).

[16] U. Gursoy, D. Kharzeev, K. Rajagopal, Phys. Rev. C 89, 054905 (2014).

[17] A. Amato et al., Phys. Rev. Lett. 111, 172001 (2013).

[18] V. Greco, C.M. Ko, P. Levai, Phys. Rev. Lett. 90, 202302 (2003).

[19] M. Cacciari et al., JHEP 10, 137 (2012).

[20] P. Bozek, I. Wyskiel, Phys. Rev. C 81, 054902 (2010).

[21] F. Becattini et al., Eur. Phys. J. C 75, 406 (2015), [Erratum: Eur.Phys.J.C 78, 354 (2018)].

[22] L. Oliva, S. Plumari, V. Greco, JHEP 05, 034 (2021). 